\title{
Magnetic Vacancy Percolation in Dilute Antiferromagnets
}

\author{
W. C. Barber, F. Ye, and D. P. Belanger \\ Department of Physics, University of California, \\ Santa Cruz, California 95064 \\ J. A. Fernandez-Baca \\ Solid State Division, Oak Ridge National Laboratory, \\ Oak Ridge, Tennessee 37831
}

(Dated: November 10, 2018)

\begin{abstract}
Neutron scattering experiments at the magnetic vacancy percolation threshold concentration, $x_{v}$, using the random-field Ising crystal $\mathrm{Fe}_{0.76} \mathrm{Zn}_{0.24} \mathrm{~F}_{2}$, show stability of the transition to long-range order up to fields $H=6.5 \mathrm{~T}$. The observation of the stable long-range order corroborates the sharp boundary observed in computer simulations at $x_{v}$ separating equilibrium critical scattering behavior at high magnetic concentration from low concentration hysteretic behavior. Low temperature $H>0$ scattering line shapes exhibit the dependence on the scattering wavevector expected for percolation threshold fractal structures.

PACS numbers:
\end{abstract}

The dilute, anisotropic antiferromagnet $\mathrm{Fe}_{\mathrm{x}} \mathrm{Zn}_{1-\mathrm{x}} \mathrm{F}_{2}$ is an extensively studied prototype of the three dimensional $(d=3)$ random-field Ising model (RFIM) [1]. As a result of the magnetic vacancies, the magnetic moment is not uniform and this allows a strong coupling to to an external magnetic field applied along the spin-ordering direction. This constitutes the mechanism for the generation of random fields [2]. It was shown that such a system is in the same universality class as a pure Ising magnet with random fields imposed [3]. Settling the question of universality of the phase transition does not, however, address the effect of vacancies on microscopic domain formation, which can mask the phase transition in scattering experiments. Such microdomain formation, which occurs since domain walls can take advantage energetically of the vacancies, needs to be well understood in order to properly interpret the RFIM behavior of dilute magnets.

For many years controversy surrounded the interpretation of neutron scattering experiments [1] on the RFIM critical behavior of dilute anisotropic antiferromagnets in external magnetic fields, $H$, particularly $\mathrm{Fe}_{\mathrm{x}} \mathrm{Zn}_{1-\mathrm{x}} \mathrm{F}_{2}$ and its less anisotropic isomorph $\mathrm{Mn}_{\mathrm{x}} \mathrm{Zn}_{1-\mathrm{x}} \mathrm{F}_{2}$. All of these studies, regardless of whether traditional scaling or various phenomenological models were used in the interpretations, were done at concentrations $x \leq 0.75$ 田, 5 . This was natural since the strength of the random field increases with dilution and available field strengths required high vacancy concentrations to readily create suitably strong random fields. It was, of course, realized that no ordering would take place for magnetic concentration below the magnetic percolation threshold concentration, $x_{p}=0.246$. The magnetic vacancy percolation threshold concentration occurs at $x_{v}=1-x_{p}=0.754$. Below this concentration, vacancies form a cluster that spans the crystal. The significant role of magnetic vacancy percolation in the formation of microdomains was not fully appreciated until recently [6] and, prior to that, it was widely assumed that microdomain formation was an intrinsic property of the RFIM as realized in dilute antiferromagnets. The microdomain structures for small $x$ have been studied extensively [7, 8]. It has recently been shown that these structures play a crucial role in exchange-bias structures important to magnetic recording technology [9, 10]. Microscopic domain structure, for which the characteristic length scale is small compared to the instrumental resolution, masks the neutron scattering critical behavior for two reasons. First, the scattering contribution from microscopic domains is superimposed on the scattering from thermal fluctuations, making it futile to separate the two. Second, there is a concomitant decrease in the Bragg scattering, which consequently no longer represents the strength of the RFIM order parameter. This has been particularly frustrating, since characterization of the RFIM transition is important in light of the present disagreements between simulations and experiments [6].

The critical behavior of $\mathrm{Fe}_{0.93} \mathrm{Zn}_{0.07} \mathrm{~F}_{2}$ using neutron scattering techniques provided evidence 11 that microscopic domains could be avoided altogether by doing measurements at high magnetic concentrations, although very high quality crystals and high fields are required. Further experiments have been done using $\mathrm{Fe}_{0.85} \mathrm{Zn}_{0.15} \mathrm{~F}_{2}$ and $\mathrm{Fe}_{0.87} \mathrm{Zn}_{0.13} \mathrm{~F}_{2}$ [12, 13. These experiments are providing the avenue for a complete experimental characterization of the RFIM universal critical behavior. It has become quite clear that the behavior at large $x$ is quite distinct from that at low $x$ which exhibits microdomain structure. Computer simulations [6] were done to model the behavior of the formation of microdomains and longrange order in $\mathrm{Fe}_{\mathrm{x}} \mathrm{Zn}_{1-\mathrm{x}} \mathrm{F}_{2}$ in an attempt to understand how the behavior crosses from one type of behavior to the other. It is suggested by these simulations that lowtemperature metastability and microscopic domain for- 
mation vanish abruptly above $x=0.76$, which closely coincides with the concentration of the magnetic vacancy percolation threshold concentration, $x_{v}=0.754$. Apparently, the percolating lattice of vacancies results in the instability of long-range order below the transition. In previous experiments, little attention has been paid to the percolation of magnetic vacancies. In light of its importance to the understanding of the RFIM we were motivated to investigate the scattering in $\mathrm{Fe}_{0.76} \mathrm{Zn}_{0.24} \mathrm{~F}_{2}$, which is very close to the critical concentration $x_{v}$. The concentration was determined using density measurements and the concentration gradient of a few tenths of a percent was determined using room temperature birefringence techniques [14].

Considerable focus has been given to the study of behavior near the complementary threshold concentration for magnetic percolation, $x_{p}=1-x_{v}$, using neutron scattering, specific heat, linear birefringence, magnetization and ac susceptibility techniques 115, 16, 17, 18, 19 . The value $x_{p}=0.246$ is based on a calculation including only the dominant $J_{2}$ interaction [20]. However, for concentrations close to $x_{p}$, the system is extremely sensitive to very weak interactions that are insignificant away from $x_{p}$. Spin-glass-like behavior at $H=0$ has been well characterized. Even far above $x_{p}$, large fields cause a crossover from the low-field microdomaindominated random-field behavior to the spin-glass-like behavior 21, 22, 23, 24, 25, 26.

The neutron scattering experiments were performed at the Oak Ridge National Laboratory High Flux Isotope Reactor using a double-axis spectrometer configuration. The beam was horizontally collimated to $20 \mathrm{~min}$ of arc before and after the sample and $48 \mathrm{~min}$ of arc before the monochromator. The neutron energy was either $13.7 \mathrm{meV}$ on the HB1 spectrometer or $14.7 \mathrm{meV}$ on the HB1A spectrometer. Higher energy neutrons were eliminated using pyrolytic graphite filters. Most of the data were taken with transverse scans about the (100) antiferromagnetic Bragg point. The $\mathrm{Fe}_{0.76} \mathrm{Zn}_{0.24} \mathrm{~F}_{2}$ crystal has an irregular shape approximately $4 \times 5 \times 10 \mathrm{~mm}$. It has a resolution limited Bragg peak, but very small secondary peaks appear for $q>0$ in the low temperature scans. Near the transition, these tiny peaks are not evident. All the data used in the analysis of the line shapes at low temperatures are on the $q<0$ side of the Bragg peak, where no hint of any secondary peaks are observed. The thermometry was based on a commercially calibrated carbon thermometer. Two primary thermal cycling procedures that are often employed to investigate hysteresis in the RFIM include: 1) cooling in the absence of a field, raising the field and warming through the transitions (ZFC); and 2) cooling in the field (FC).

Figure 1 shows scattering intensity vs. q, in reciprocal lattice units (rlu), at $H=3$ and 5 T close to the transition temperatures $T_{c}=61.6$ and $60.4 \mathrm{~K}$, respectively. The transition at $H=0$ is at $63.2 \mathrm{~K}$. Whereas the criti-

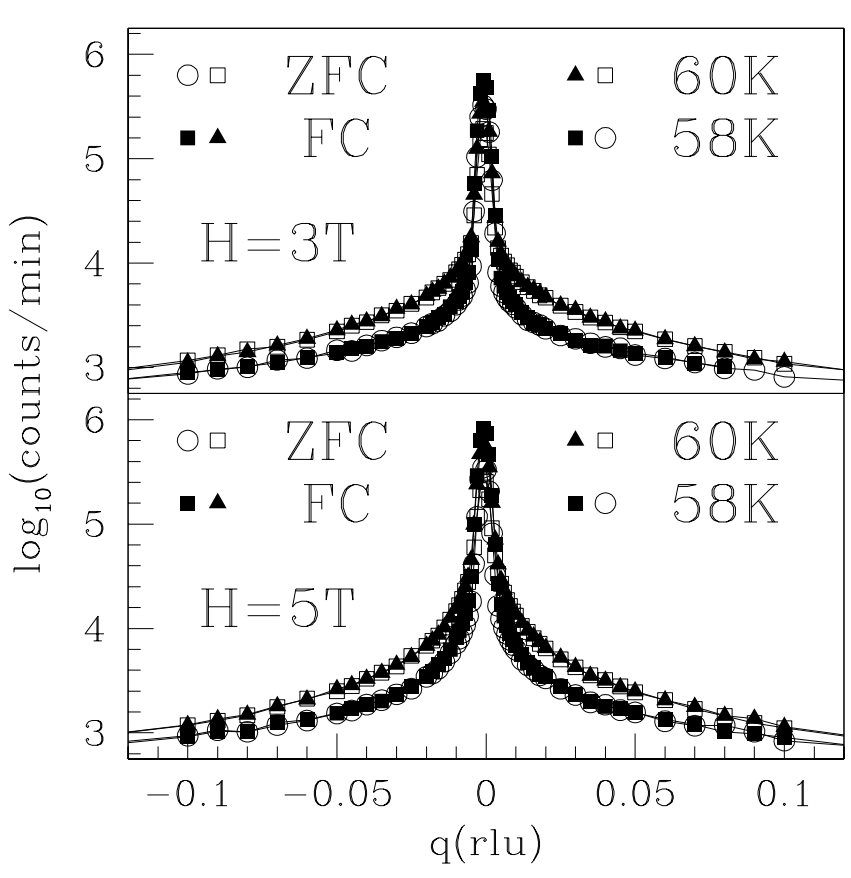

FIG. 1: ZFC and FC neutron scattering at $H=3$ and $5 T$ below but close to the transition temperature. The contributions include a constant background, critical scattering which is seen to increase as the temperature approaches $\left(T_{c}(H)\right)$, and a Bragg peak due to long range order.

cal scattering from samples with $x<x_{v}$ exhibits strong hysteresis, it is clear that the $|q|>0$ critical scattering shown in Fig. 1 is free from hysteresis and that indicates there is no microscopic domain structure frozen in upon FC.

For experiments free of extinction effects, the magnetic Bragg scattering intensity is expected to follow the power law behavior

$$
I=M_{s}^{2} \sim|t|^{2 \beta}
$$

where $M_{s}$ is the staggered magnetization and $\beta \approx 0.35$ for the random-exchange model and $\beta \approx 0.16$ the RFIM [13]. However, neutron scattering in high-quality bulk crystals can suffer from severe extinction; the beam is depleted of neutrons that satisfy the Bragg condition and the scattering intensity is therefore saturated and cannot exhibit the correct $T$ dependence. The extinction effects usually preclude determination of a reliable value of $\beta$, the critical exponent for the staggered magnetization, from an analysis of the neutron scattering data in very high quality bulk crystals. The Bragg scattering does show hysteresis, which indicates incomplete FC ordering on very long length scales, relative to the instrumental resolution. Such hysteresis occurs for all $x$ and is likely a consequence of the slow activated dynamics 27, 28, 29, 30] of the RFIM very close to $T_{c}(H)$.

The ZFC Bragg intensity, corrected for the background 


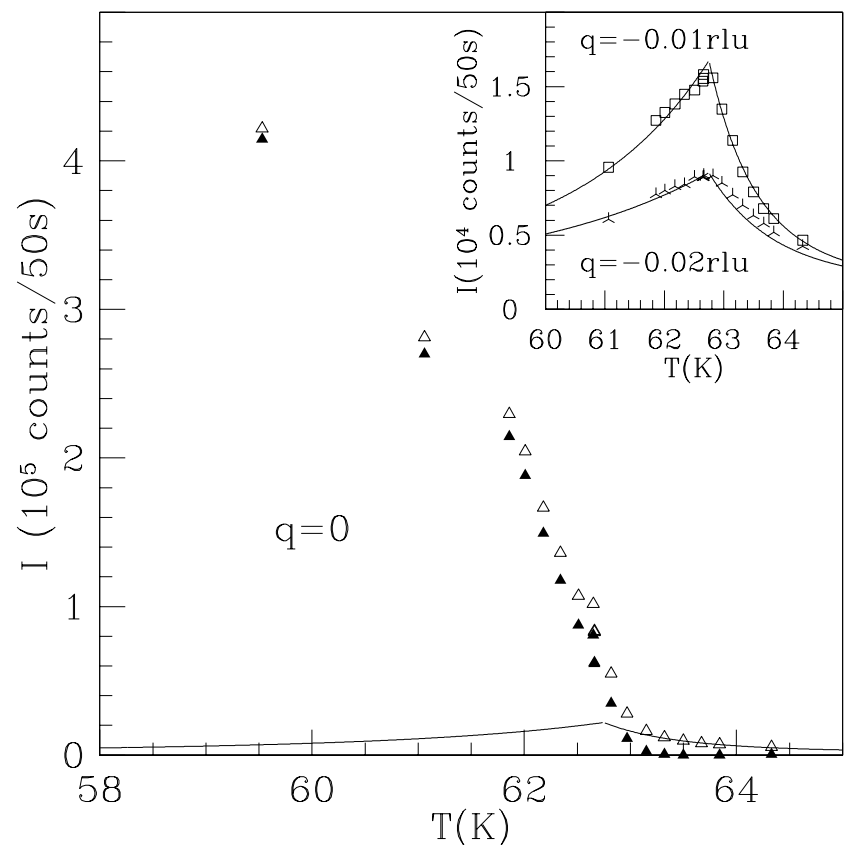

FIG. 2: The ZFC antiferromagnetic Bragg peak for $H=3 T$. The contribution at $q=0$ due to critical fluctuations is subtracted from the data represented by the open symbols to give the corrected intensities represented by the solid symbols.

determined at high $T$, is shown in Fig. 2 vs. $T$ for $H=3 \mathrm{~T}$. The inset of Fig. 2 shows the critical scattering at small $q$, but well outside the transverse instrumental resolution where the Bragg intensity is negligible. The scattering line shapes in this sample are complicated by admixture of critical scattering and contributions from the vacancy lattice because of the proximity of this sample to $x_{v}$, as will be discussed. Hence, we could not confidently analyze in detail the critical scattering line shape. Nevertheless, we obtained an approximate accounting of the critical scattering intensity by taking a squared-Lorentzian line shape folded with the appropriate resolution correction. An overall amplitude had to be chosen for the fit shown in the inset. This same amplitude was applied to the $q=0$ case and the resulting curve is shown in the main part of Fig. 2 as the solid curves. Subtracting this from the raw data (open symbols) yields the corrected Bragg scattering data (filled symbols). Taking into account the concentration gradient rounding of a few tenths of a percent, it is quite apparent that the Bragg intensity data approach $T_{c}(H)$ with a steep slope, in contrast with scattering experiments [31] with $x<x_{v}$, where the slope is nearly zero. Although we cannot analyze the data according to Eq. 1 to obtain $\beta$ because of extinction, we may still conclude from the shape of the Bragg intensity vs. $\mathrm{T}$ that this sample does not form microscopic domain structure. This is consistent with the lack of hysteresis in the critical scattering shown in Fig. 1.

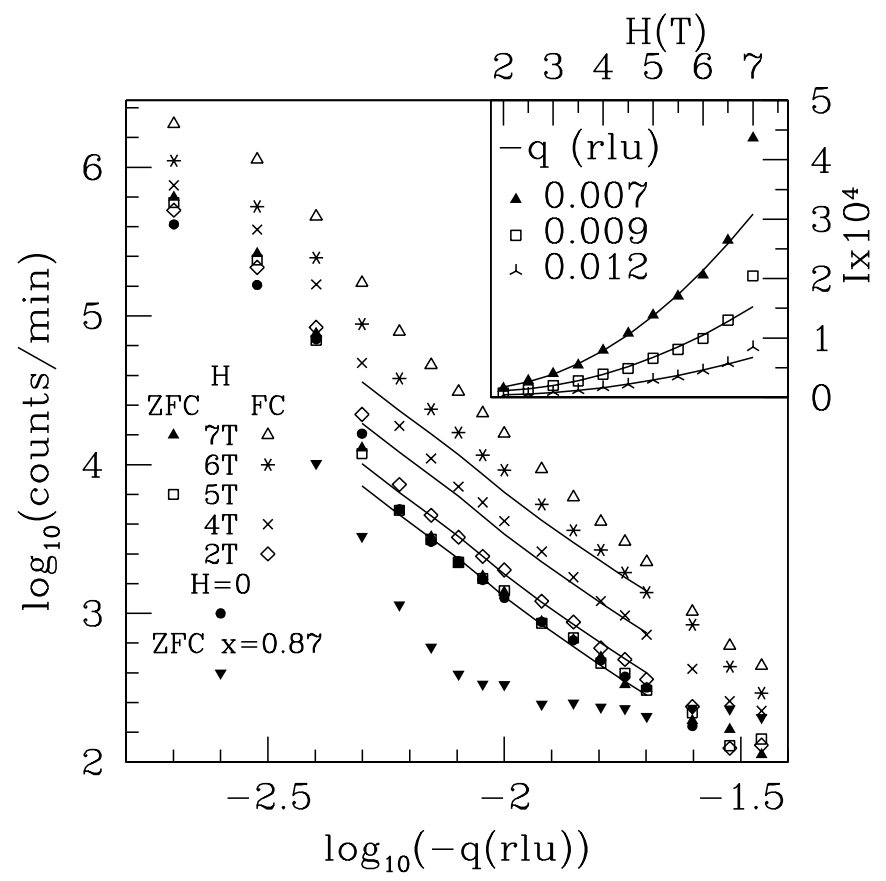

FIG. 3: The logarithm of the neutron scattering intensity vs. the logarithm of $q$ for $H=0 T$ and for ZFC and FC up to $H=7 T$ at $T=20 K$, well below $T_{c}(H)$. The background determined at large $q$ has been subtracted. Only a few sets of data are shown for clarity. All ZFC data lie on the same curve, but only $H=5$ and $7 \mathrm{~T}$ data are shown.

At the percolation threshold concentrations, magnetic sites or magnetic vacancy sites form fractal structures. In either case, scattering from the fractal structure will exhibit a power law behavior 32, 33]

$$
I_{f} \sim q^{-2.53}
$$

The only difference is that in the case of magnetic vacancies there is also a Bragg scattering peak from the average $M_{s}$. With magnetic site percolation, the average $M_{s}$ is zero at the threshold. Since we believe the $x=0.76$ crystal is close to $x_{v}$, we plotted the logarithm of the scattering intensity vs. the logarithm of $-q$ for $q<0$ in Fig. 3. Only some of the scans are shown; data were taken for $H=0$ and for $2 T<H<7 \mathrm{~T}$ in steps of 0.5 T. Several interesting features are evident.

As shown explicitly for two fields, $H=5$ and $7 \mathrm{~T}$ in Fig. 3, the ZFC line shapes, for $T=29 \mathrm{~K}$ or less, are all identical with the $H=0$ line shape and are the lowest in intensity. For comparison, a line with a fractal exponent of 2.53 for three dimension, with the spectrometer resolution folded in, is plotted in the graph with the amplitude adjusted to fit the ZFC intensity data. It is clear that the ZFC scattering line shapes for $x=0.76$ follow Eq. 2 quite well. To contrast this behavior, we show in Fig. 3 similar data for a sample with concentration of $x \approx 0.87[12]$, indicated by the solid triangles, 
for $T=58.5 \mathrm{~K}$, only $3.4 \mathrm{~K}$ below the transition. The scattering for this concentration, well above $x_{v}$, shows little evidence of scattering outside the Bragg region as expected since the vacancies do not form large fractal structures at this concentration. The behavior for ZFC shown in Fig. 3 suggests strongly that, for $x=0.76$, the scattering is indeed from the vacancy percolation fractal structure under the ZFC procedure.

The FC data for $x=0.76$ increase in intensity with the applied field. We compare the data to Eq. 2 by adjusting the amplitude to fit the data at $q=10^{-1.7} \mathrm{rlu}$. It is quite clear that for $H>0$ the line shapes deviate strongly from the behavior in Eq. 2, more so as the field increases. The inset of Fig. 3 shows the deviations of the intensities from the curves representing Eq. 2 at $q=-0.007$, -0.009 , and -0.012 rlu as a function of the applied field. The deviations for $H \leq 6.5 \mathrm{~T}$ increase smoothly with the field. Two possible sources exist for the excess scattering. One is the relief of extinction. This has been observed for neutron Bragg scattering in the RFIM experiments on bulk crystals [1], but not for scattering outside the Bragg region. The other possibility, perhaps more significant, is the scattering from domains, which coexist with antiferromagnetic long-range order, that increase in number with increasing applied field. Since the total scattering in FC comes from sources in addition to that of vacancy sites, it is difficult to analyze in detail.

The difference between FC intensities and Eq. 2 at $H=7 \mathrm{~T}$ deviate strongly from the smooth curves describing the data for $H \leq 6.5 \mathrm{~T}$, as shown in the inset of Fig. 3. This qualitatively new behavior most likely represents a breakdown of the antiferromagnetic long-range structure for $H>6.5 \mathrm{~T}$. In such a case many more domains are introduced into the system, resulting in much more scattering intensity. This field is consistent with the increasing field at which spin-glass-like behavior appears for samples with $x<x_{v}$, as shown in Fig. 4. Apparently, even at low temperatures, long-range antiferromagnetic order for $x=0.76$ is stable upon FC for $H<7 \mathrm{~T}$, in contrast to the behavior for $x<x_{v}$, where metastable domains dominate the scattering under the FC procedure. For $x \approx 0.87$, only a few percent above $x_{v}$, magnetization experiments [34] indicate that the transition to long-range antiferromagnetic order breaks down only for fields above $H=18 \mathrm{~T}$, nearly three times the field that causes a breakdown in the behavior of the sample with $x=0.76$, demonstrating the stability of antiferromagnetic order for $x>x_{v}$.

We have shown that the magnetic concentration range for which equilibrium random field critical scattering is observed for dilute antiferromagnets in an external field has a lower bound at $x_{v}$. Whereas our $x=0.76$ crystal shows no critical scattering hysteresis, it is quite evident for slightly smaller magnetic concentrations [5]. Our results suggest that the percolation of vacancy sites occurring for $x<x_{v}$ precipitates the formation of domains

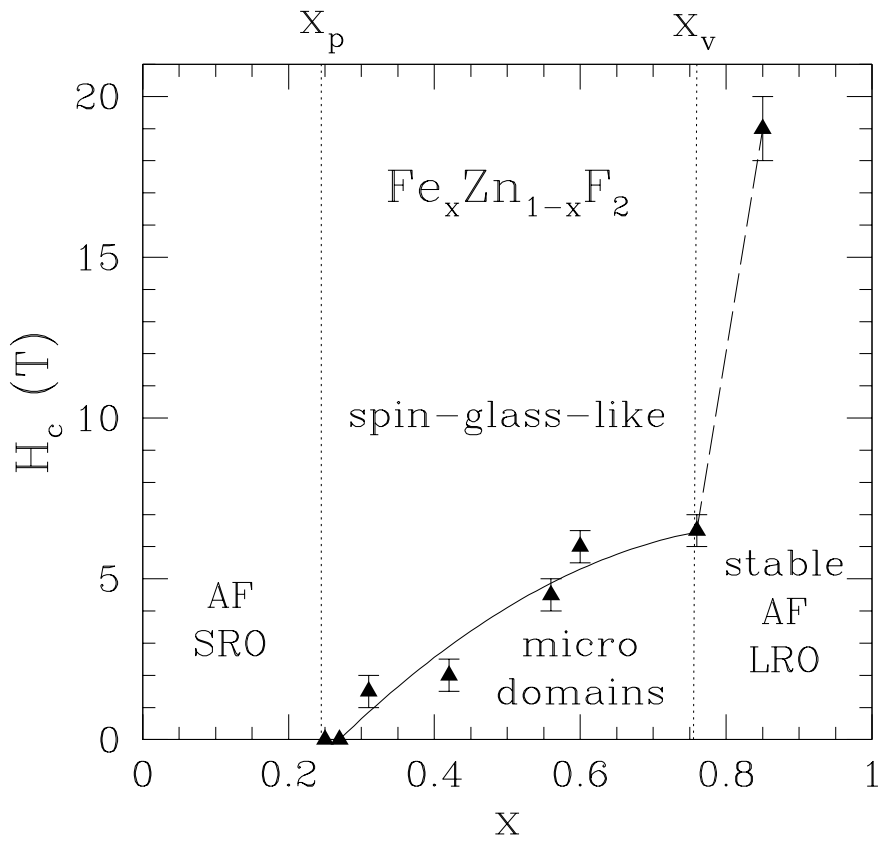

FIG. 4: The concentration dependence of the phases observed in $\mathrm{Fe}_{\mathrm{x}} \mathrm{Zn}_{1-\mathrm{x}} \mathrm{F}_{2}$. For $x<x_{p}$, the magnetic percolation threshold concentration, only antiferromagnetic short-range order is possible. For $x_{p}<x<x_{v}$, microscopic domain formation occurs at low fields and spin-glass-like behavior occurs at high fields. For $x>x_{v}$, antiferromagnetic long-range order is stable, without microscopic domain formation, to very large applied fields. Critical behavior measurements can only be reliably done for $x>x_{v}$. Data are taken from various experiments cited in the text.

below $T_{c}(H)$ in ZFC preparation as well as in FC, corroborating the conclusions drawn from simulations [6]. A theoretical connection between the one dimensional fractal geometry of vacancy sites at percolation and three dimensional domains has not been adequately explained from a theoretical perspective.

It is now evident that there are three magnetic concentration regimes in $d=3$ dilute antiferromagnets separated by the percolation threshold concentrations $x_{p}$ and $x_{v}$. For $x>x_{v}$, long-range antiferromagnetic order is stable up to very large magnetic fields. For $x_{p}<x<x_{v}$ the system is unstable. At low fields, the formation of microdomain structure takes place upon $\mathrm{FC}$ for all $T<T_{c}(H)$ and upon ZFC close to $T_{c}(H)$. A spin-glasslike phase forms at high field. Below $x_{p}$, there can be no long-range magnetic order. It is the geometry of the lattice in question which defines the location of these boundaries, and although we study one particular magnetic lattice type, the body centered tetragonal structure of $\mathrm{Fe}_{\mathrm{x}} \mathrm{Zn}_{1-\mathrm{x}} \mathrm{F}_{2}$, our results should apply more generally to dilute magnets in an applied field.

Interestingly, the specific heat behavior is not dependent in an obvious way on the concentration. Similar 
hysteresis upon $\mathrm{FC}$ and $\mathrm{ZFC}$ is observed [1] very close to $T_{c}(H)$ for concentrations above and below $x_{v}$. No specific heat hysteresis is observed at low $T$. The contrast between the relative insensitivity of the specific heat techniques with the extreme sensitivity of the scattering techniques is certainly due to the greater dependence of the scattering on long length correlations that are greatly affected by domain formation. The hysteresis in the case of specific heat is related to the activated dynamics very close to $T_{c}(H)$ that affects the behavior at all $x$ and not domain formation, which only occurs for $x<x_{v}$.

From the results of this investigation, we conclude that studies of the random-field phase transition should be conducted with magnetic concentrations greater than $x_{v}$. It is advantageous to use concentrations not too much greater than this to maximize the random-fields for available applied fields. However, if the concentration is too close to $x_{v}$, one must take into account scattering from the magnetic vacancy percolation cluster. Recent experiments at $x=0.87$ indicate that, at this concentration, such scattering is negligible [12, 13].

This work was funded by Department of Energy Grant No. DE-FG03-87ER45324 and by the Oak Ridge National Laboratory, which is managed by UT-Battelle, LLC, for the U.S. Dept. of Energy under contract DEAC05-00OR22725.

[1] D.P. Belanger, Brazilian J. of Phys. 30, 682 (2000) and references therein.

[2] S. Fishman and A. Aharony, J. Phys. C 12, L729 (1979).

[3] J.L. Cardy, Phys. Rev. B 29, 505 (1984).

[4] D. P. Belanger , S. M. Rezende, A. R. King, and V. Jaccarino, J. Appl. Phys. 57, 3294 (1985).

[5] J. P. Hill, Q. Feng, R. J. Birgeneau and T. R. Thurston, Phys. Rev. Lett. 70, 3655 (1993), although the nominal concentration is $x=0.75$ the actual concentration may be slightly less, judging from the $H=0$ transition temperature 35].

[6] W.C. Barber and D.P. Belanger, J. Appl. Phys. 87, 7049 (2000).

[7] S.-J. Han, D.P. Belanger , W. Kleemann and U. Nowak, Phys. Rev. B 45, 9728 (1992).

[8] D.P. Belanger, V. Jaccarino, A.R. King, and R.M. Nicklow, Phys. Rev. Lett. 59, 930, (1987).

[9] P. Miltnyi, M. Gierlings, J. Keller, B. Beschoten, G. Gntherodt, and U. Nowak and K. D. Usadel, Phys. Rev. Lett. 84, 4224 (2000).

[10] S. Zhang, D. V. Dimitrov, G. C. Hadjipanayis, J. W. Cai, and C. L. Chien, J. Magn. Magn. Mater. 198-199, 468 (1999).

[11] Z. Slanic, D.P. Belanger, J.A. Fernandez-Baca, Phys. Rev. Lett. 82, 426, (1999).

[12] F. Ye, M. Matsuda, S. Katano, H. Yoshizawa, J. A. Fernandez-Baca, D. P. Belanger, unpublished.

[13] F. Ye, L. Zhou, S. Larochelle, L. Lu, D. P. Belanger, M. Greven, and D. Lederman, Phys. Rev. Letters 89, 157202 (2002).

[14] A. R. King, I. B. Ferreira, V. Jaccarino, and D. P. Belanger, Phys. Rev. B 37, 219 (1988).

[15] D. P. Belanger and H. Yoshizawa, Phys. Rev. B 47, 5051 (1993).

[16] F. C. Montenegro, M. D. Coutinho-Filho, and S. M. Rezende, Europhys. Lett. 8, 382 (1989).

[17] E. P. Barbosa, E. P. Raposo and M. D. Coutinho-Filho, J. Appl. Phys. 87, 6531 (2000).

[18] W. C. Barber and D. P. Belanger, Phys. Rev. B 61, 8960 (2000).

[19] K. Jonason , C. Djurberg, P. Nordblad, and D. P. Belanger, Phys. Rev. B bf 56, 5404 (1997).

[20] M. F. Sykes and J. W. Essam, Phys. Rev. 133, A310 (1964).

[21] F.C. Montenegro, A.R. King, V. Jaccarino, S-J. Han and D.P. Belanger, Phys. Rev. B 44, 2155 (1991).

[22] D. P. Belanger , Wm. E. Murray, Jr., F. C. Montenegro, A. R. King, V. Jaccarino and R. W. Erwin, Phys. Rev. B 44, 2161 (1991).

[23] F. C. Montenegro, K. A. Lima, M. S. Torikachvili, and A. H. Lacerda, Mater. Sci. Forum 302-303, 371 (1999).

[24] F. C. Montenegro, K. A. Lima, M. S. Torikachvili, and A. H. Lacerda, J. Magn. Magn. Mater. 177-181, 1451998.

[25] A. Rosales-Rivera, J. M. Ferreira, and F. C. Montenegro, Europhys. Lett. 50, 2642000.

[26] J. Satooka, H. Aruga Katori, A. Tobo and K. Katsumata, Phys. Rev. Lett. 81, 709 (1998).

[27] D. S. Fisher, Phys. Rev. Lett. 56, 416 (1986).

[28] A. R. King, J. A. Mydosh and V. Jaccarino, Phys. Rev. Lett. 56, 2525 (1986).

[29] A. E. Nash, A. R. King and V. Jaccarino, Phys. Rev. B 43, 1272 (1991).

[30] Ch. Binek, S. Kuttler and W. Kleemann, Phys. Rev. Lett. 75, 2412 (1995).

[31] D. P. Belanger, J. Wang, Z. Slanic, S-J. Han, R. M. Nicklow, M. Lui, C. A. Ramos and D. Lederman, Phys. Rev. B 54, 3420 (1996).

[32] D. Stauffer and A. Aharony, Introduction to Percolation Theory, 2nd Ed., Francis and Taylor, (London), 1994.

[33] H. Ikeda, K. Iwasa, J.A. Fernandez-Baca, and R.M. Nicklow, Physica B 213, 146 (1995).

[34] T. Sakon, A. Awaji, M. Motokawa, and D. P. Belanger, J. Phys. Soc. Japan 71, 411 (2002).

[35] D. P. Belanger , A. R. King, F. Borsa, and V. Jaccarino, J. Magn. Magn. Mat. 15-18, 807-808 (1980). 